\title{
RELATION BETWEEN SCHISTOSOME PAST INFECTION AND METABOLIC SYNDROME
}

\author{
By \\ SABAH M. A. MOHAMED', MAHA SABER ${ }^{1}$, AFAF A. TAHA', \\ HISHAM S. ROSHDY 2 and NOHA E. SHAHIEN ${ }^{3}$ \\ Departments of Medical Parasitology ${ }^{1}$, Cardiology ${ }^{2}$, and Tropical Medicine ${ }^{3}$, Faculty \\ of Medicine, Zagazig University ( ${ }^{*}$ Correspondence:drafaf343@yahoo.com)

\section{Abstract}

Schistosome antigens modulate host metabolic profiles in experimental animals. The effects of previous schistosome infection (PSI) and the development of metabolic syndrome remain unknown in humans. This study evaluated previous schistosome infection (PSI) related to metabolic syndrome (MS). A total of 547 participants aged $\geq 40$ years from rural areas of Zagazig district were enrolled. Of them, 269 patients with PSI and 305 normal served as controls. For all participants blood pressure, height, body weight and waist circumstance (WC) were measured. Blood samples were examined biochemically to determine triglyceride (TG), fasting blood glucose (FBG) and high-density lipoprotein cholesterol (HDL-C). Associations between PSI and MS were evaluated using logistic regression. Patients with PSI had significantly lower levels of body mass index (BMI), WC, TG, insignificantly low levels of fasting blood glucose (FBG) and significantly higher levels of high-density lipoprotein cholesterol (HDL-C) compared with controls. Prevalence of MS in PSI was significantly low than controls (32.7\% vs. $42.3 \%$ respectively). PSI significantly associated with low prevalence of metabolic syndrome and its components, including central obesity, hypertension, hyperglycemia, hypertriglyceridemia and low HDL-C. Potential long-term effects of PSI may reduce metabolic syndrome risk.

Keywords: Previous schistosome infection, Metabolic syndrome.

\section{Introduction}

Schistosomiasis poses a major public health threat in many tropical and subtropical countries. The disease is endemic in 78 countries worldwide. In 2011, at least 243 million people were infected and needed treatment (Liang et al, 2014). Metabolic syndrome is a condition that includes the presence of a cluster of risk factors specific for cardiovascular disease. A meta-analysis of longitudinal studies revealed a significantly higher risk of cardio-cerebro-vascular diseases and death in patients with MS compared with non-MS subjects (Mottillo et al, 2010). Some studies have shown an inverse association between helminth infections and inflammatory diseases, including MS, cardiovascular diseases (Sengupta, 2008).

Helminth parasites are the strongest natural inducers of type 2 inflammatory responses, and epidemiologic studies in India and rural China revealed that helminth infections inversely correlate with metabolic syndrome (Wiria et al, 2014). Some studies recently reported that the rodent nematode Nippostrongylus brasiliensis, which is sponta- neously cleared within 2 weeks of infection, improves glucose tolerance in diet-induced obese mice (Wu et al, 2011). These studies suggested that manipulation of the immune system by helminths or their molecules might be beneficial for metabolic homeostasis.

However, it remains unclear which aspects of whole-body energy metabolism were affected by the worms (Wiria et al, 2014). Schistosomiasis was regarded as both a helminthic infection and an immunological disease (Zhu et al, 2008). Schistosome antigens (SEA) was found to induce metabonomic alterations and mediate the host immune response (Wu et al, 2011), which produced a strong anti-inflammatory response to help the suppression of the development of arteriosclerosis in mice (Subramanian et al, 2013).

This study evaluated the association between the PSI and the risk of developing MS components to provide evidence for the development of new strategies for prevention and control of MS. 


\section{Materials and Methods}

Type of study: A cross-sectional study.

Study population: The present study included 574 participants aged 41-70Y, 415 male and 159 female from Internal Medicine, Tropical medicine Outpatient Clinics of Zagazig University Hospitals from June 2012 to April 2015. Participants with a history of viral hepatitis, decompensated cirrhosis, malignant tumors, kidney dysfunction, and use of statin drugs, active schistosome infection or incomplete medical records were excluded.

The study was confined to participants from rural areas in Zagazig District. All were subjected to full medical history, socioeconomic and educational classes and underwent complete clinical examination, abdominal sonography. The collected records of all participants were reviewed retrospectively. Stool samples examination by KatoKatz method to confirm all subjects were parasite free at the time of sampling.

They were divided into group with previous schistosome infection (PSI) (269 participants) and group without PSI as a control group (305 participants).

All participant's blood pressure, height, body weight and waist circumstance (WC) were measured. All participants fasted for 8$12 \mathrm{~h}$ prior to collection of $5 \mathrm{~mL}$ of venous blood on next morning. Levels of FBG were measured using the glucose oxidase method on an autoanalyzer (ADVIA-1650 chemistry system, Siemens, Munich, Germany). Hemoglobin $(\mathrm{Hb})$ levles were counted in whole-blood samples using a model T890 Coulter counter (Coulter, Miami, FL). Alanine aminotransferase (ALT), TG, HDL-C were measured using an enzymatic colorimetric method (LX-20; Beckman, Brea,CA).

Evaluation of tests: According to the International Diabetes Federation 2005 criteria (Alberti et al, 2005) MS was diagnosed as central obesity $\mathrm{WC} \geq 102 \mathrm{~cm}$ in men and $\geq$ $88 \mathrm{~cm}$ in women and any two of the following four criteria: (1) $\mathrm{BP} \geq 130 / 85 \mathrm{mmHg}$ or taking antihypertensive drugs; (2)TG $\geq 150$ $\mathrm{mg} / \mathrm{dl}$;(3) HDL-C $<40 \mathrm{mg} / \mathrm{dl}$ in men, < 50 $\mathrm{mg} / \mathrm{dl}$ in women ; (4) $\mathrm{FBG} \geq 100 \mathrm{mg} / \mathrm{dl}$ or taking hypoglycemic medications .Obesity was defined as a body mass index (BMI) of $\geq 30 \mathrm{~kg} / \mathrm{m} 2$ (Liu et al.,2002). BMI is defined as the body mass (weight) divided by the square of the body height and is universally expressed in units of $\mathrm{kg} / \mathrm{m}^{2}$, resulting from mass in kilograms and height in metres.

PSI was defined as follows: History of living in a schistosomiasis-endemic area, contact with infested water, drug treatment and a definite diagnosis of chronic schistosomal liver disease detected with B-mode ultrasonography. The criteria for an ultrasound diagnosis of chronic schistosomal liver disease involved thickened liver parenchyma, with enhanced echo intensity that displayed a grid-like distribution, dilation of the portal vein (main trunk diameter $>1.3 \mathrm{~cm}$ ), with a thickened luminal wall, and enlargement of the left lobe of the liver and atrophy of the right lobe, with the external left lobe appearing blunt and round (Richter et al, 2000)

Ethical consideration: A detailed description of the purpose and potential benefits of this study was explained to the participants prior to sample collection and a written or thumb-printed informed consent was obtained. The study was approved by the Committee of Research, Publications and Ethics of the college of Medicine, Zagazig University, Egypt.

Statistical analysis: Data were expressed as mean \& standard deviation (SD) for continuous variables and as a percentage for categorical variables using Student's t-tests for continuous variables and chi-square test for categorical variables. Correlations between PSI and MS and its components were evaluated by logistic regression analysis. A $\mathrm{P}$ value $<0.05$ was considered statistically significant. Statistical analyses were performed using the statistical software SPSS version 11.5 (SPSS Inc., Chicago, IL, USA).

\section{Results}

The mean age of the participants was $57 \pm$ 8.1 years ranging from 41 to 73 years. Four 
hundred and fifteen $(72.3 \%)$ were males and $159(27.7 \%)$ were females. Regarding the educational levels percentages in the sample it was $176(30.7 \%), 117(20.4 \%), 203$ $(35.4 \%)$ and $78(13.6 \%)$ for illiterate, basic education, secondary education and high education respectively. One hundred and thirty four $(23.3 \%)$ participant gave history of hypertension. One hundred and nine (19\%) participants gave history of diabetes mellitus. Two hundred and seventeen (37.8\%) participants were diagnosed with metabolic syndrome.

The participants were divided into two groups according to history of previous Schistosoma infection into a control group $(n=305)$ and PSI $(n=269)$ group, both groups were matched in age, sex and educational level. Patients with PSI had significant $(\mathrm{P}<0.05)$ lower history of diabetes $(15.2 \%$ \& $22.3 \%$ respectively) and lower history of hypertension $(21.9 \%$ \& $24.6 \%$ respectively) without significant difference $(\mathrm{P}>0.05)$ when compared with control group.

There was significantly $(\mathrm{P}<0.05)$ lower BMI and WC in PSI group $(29.7 \pm 6.19 \&$ $104.5 \pm 16.8$ vs. $31.4 \pm 6.92 \& 109 \pm 19.12$ respectively) compared with the control group. Individuals with PSI had highly significant $(\mathrm{P}<0.001)$ lower TG compared with control group (120.4 $\pm 29.59 \& 133 \pm 30.52$ respectively). FBG levels were lower in PSI group than in the control ones $(105.8 \pm 23.1 \&$ $107.8 \pm 26.36$ respectively) without significance $(\mathrm{P}>0.05)$, and significantly higher $(\mathrm{P}<0.001)$ HDL-C $(53 \pm 10.23 \& 50.4 \pm 10.17$ respectively) compared with controls. Also, individuals with PSI had significant lower

Table 1: Demographic criteria of different groups

\begin{tabular}{|c|c|c|c|c|c|}
\hline \multicolumn{2}{|c|}{ Groups } & Control $(\mathrm{n}=305)$ & With PSI $(n=269)$ & Total $(n=574)$ & $\mathrm{P}$ \\
\hline \multirow{2}{*}{ Sex : } & Male & $221(725 \%)$ & $\frac{1 \times(70)}{194(721 \%)}$ & $\frac{1 \times(7)}{415(723 \%)}$ & \multirow{2}{*}{0.928} \\
\hline & Female & $84(27.5 \%)$ & $75(27.9 \%)$ & $159(27.7 \%)$ & \\
\hline \multirow[t]{4}{*}{ Education } & Illiterate & $94(30.8 \%)$ & $82(30.5 \%)$ & $176(30.7 \%)$ & \multirow{4}{*}{0.787} \\
\hline & Basic & $62(20.3 \%)$ & $55(20.4 \%)$ & $117(20.4 \%)$ & \\
\hline & Secondary & $109(35.7 \%)$ & $94(34.9 \%)$ & $203(35.4 \%)$ & \\
\hline & High & $40(13.1 \%)$ & $38(14.1 \%)$ & $78(13.6 \%)$ & \\
\hline \multicolumn{2}{|c|}{ History of Hypertension } & $75(24.6 \%)$ & $59(21.9 \%)$ & $134(23.3 \%)$ & 0.453 \\
\hline \multicolumn{2}{|c|}{ History of Diabetes mellitus } & $68(22.3 \%)$ & $41(15.2 \%)$ & $109(19 \%)$ & 0.032 \\
\hline
\end{tabular}

systolic and diastolic blood pressure $(110.4 \pm 22.76 \& 76.2 \pm 8.65$ vs. $126.6 \pm 11.78$ \& 78.1 \pm 7.47 respectively) compared with controls. Individuals with PSI had nonsignificant $(\mathrm{P}>0.05)$ lower level of ALT and higher Hb. level $(21.9 \pm 4.92 \& 12.8 \pm 1.76$ vs. $22.3 \pm 5.16 \& 12.5 \pm 1.47$ respectively) compared with controls.

The prevalence of metabolic syndrome was significantly $(\mathrm{P}<0.05)$ lower in the PSI group compared with the control group (32.7\% vs $42.3 \%$ respectively). Logistic regression analysis revealed statistically significant relative risk reduction in MS in PSI when compared with control group (odds ratio $[\mathrm{OR}]=0.633,95 \%$ confidence interval [CI]: $0.472-0.933, \mathrm{P}<0001)$ and its components including central obesity $(\mathrm{OR}=1.728$., 95\% CI: $1.255-2.378, \mathrm{P}<005)$, hypertriglyceridemia $(\mathrm{OR}=1.027,95 \%$ CI:1.013-1.042 , $\mathrm{P}<0001)$, low HDL-C (OR=0.899, 95\% CI: 0.861-0.940, $\mathrm{P}<0001$ ), hypertension (OR $=1.054,95 \%$ CI: $1.020-1.146, \mathrm{P}<0001)$ and hyperglycaemia (OR=1.057, 95\% CI: 1.037 1.078, $\mathrm{P}=0001)$. More evaluation of the most associated components of the MS with each of both groups using forward binary logistic regression analysis showed that: in control group: the most associated components were: female sex, reduced HDL, history of hypertension and increased TG, WC and FBS. In PSI group: the most associated components were: history of hypertension reduced HDL and increased BMI, TG, diastolic BP \& FBS.

Details were given in tables $(1,2,3,4$ \& $5)$. 
Table 2: Demographic and biochemical parameters of PSI and control

\begin{tabular}{|c|c|c|c|}
\hline & Control $(n=305)$ & With PSI $(n=269)$ & \multirow{2}{*}{$\mathrm{P}$} \\
\hline & mean \pm SD & mean $\pm \mathrm{SD}$ & \\
\hline Age (years) & $57.9 \pm 9.34$ & $56.7 \pm 6.49$ & 0.095 \\
\hline Weight( Kgm) & $79.5 \pm 19.99$ & $76.0 \pm 18.61$ & 0.28 \\
\hline Height $(\mathrm{cm})$ & $158.7 \pm 10.05$ & $159.4 \pm 10.32$ & 0.373 \\
\hline BMI $\left(\mathrm{kg} / \mathrm{m}^{2}\right)$ & $31.4 \pm 6.92$ & $29.7 \pm 6.19$ & 0.002 \\
\hline $\mathrm{WC}(\mathrm{cm})$ & $109.0 \pm 19.12$ & $104.5 \pm 16.80$ & 0.003 \\
\hline TG (mg/dl) & $133.0 \pm 30.52$ & $120.4 \pm 29.59$ & $<0.001$ \\
\hline HDL (mg/dl) & $50.4 \pm 10.17$ & $53.0 \pm 10.23$ & 0.002 \\
\hline FBS (mg/dl) & $107.8 \pm 26.36$ & $105.9 \pm 23.10$ & 0.352 \\
\hline SBP (mmHg) & $126.6 \pm 11.78$ & $110.4 \pm 22.76$ & $<0.001$ \\
\hline DBP (mmHg) & $78.1 \pm 7.47$ & $76.2 \pm 8.65$ & 0.005 \\
\hline $\mathrm{Hb}(\mathrm{gm} / \mathrm{dl})$ & $12.5 \pm 1.47$ & $12.8 \pm 1.76$ & 0.380 \\
\hline ALT & $22.3 \pm 5.16$ & $21.9 \pm 4.92$ & 0.339 \\
\hline
\end{tabular}

Abbreviations: SBP, systolic blood pressure; DBP, diastolic blood pressure

Table 3: Relation between MS and PSI

\begin{tabular}{|c|c|c|c|c|}
\hline \multirow{2}{*}{ Metabolic syndrome } & \multicolumn{3}{|c|}{ Groups } & \multirow{2}{*}{ P } \\
\cline { 2 - 4 } & Control $(\mathrm{n}=305)$ & PSI $(\mathrm{n}=269)$ & Total $(\mathrm{n}=574)$ & \multirow{3}{*}{00.018} \\
\cline { 2 - 4 } & No $(\%)$ & No $(\%)$ & No $(\%)$ & \\
\hline No Metabolic syndrome & $176(57.7 \%)$ & $181(67.3 \%)$ & $357(62.2 \%)$ & \\
\hline Metabolic syndrome & $129(42.3 \%)$ & $88(32.7 \%)$ & $217(37.8 \%)$ & \\
\hline
\end{tabular}

Table: 4: Relation between PSI and risk of MS

\begin{tabular}{|l|c|c|c|c|}
\hline & & \multicolumn{2}{|c|}{$95.0 \%$ C.I. } & \multirow{2}{*}{ MS and its components } \\
\cline { 3 - 4 } \multicolumn{1}{|c|}{ Odds ratio } & Lower & Upper & P \\
\hline Metabolic syndrome & 0.663 & 0.472 & 0.933 & 0.002 \\
\hline Central obesity & 1.728 & 1.255 & 2.378 & 0.001 \\
\hline Hypertriglyceridemia & 1.027 & 1.013 & 1.042 & 0.000 \\
\hline Low HDL- C & 0.899 & 0.861 & 0.940 & 0.000 \\
\hline Hypertension & 1.054 & 1.020 & 1.146 & 0.000 \\
\hline Hyperglycemia & 1.057 & 1.037 & 1.078 & 0.000 \\
\hline
\end{tabular}

\section{Discussion}

The clinical trials showed that infection with Trichuris suis, Necator americanus, Hymenolepis diminuta were effective for the treatment of Cohn's disease (Reddy and Fried, 2007; 2009; Melon et al, 2010). Also, the specific immune pathways, such as modified type- 2 responses, induced by helminthes were implicated in protective host responses to homoeostatic perturbations as metabolic dysfunction and atherosclerosis (von Moltke and Locksley, 2014). The present study showed a significantly $(\mathrm{P}<0.02)$ lower prevalence of MS (32.7\%) in the PSI group than that in the control group (42.3\%).

Also, Chen et al. (2013) reported that the prevalence of metabolic syndrome was lower in the PSI patients than controls (35\% and $65 \%$ respectively). Another study found that the PSI significantly correlated with lower prevalence of the metabolic syndrome (Shen et al, 2015).

The present study showed that the patients with PSI had significantly $(\mathrm{P}<0.02)$ higher HDL-C levels compared with the controls $(53 \pm 10.2 \& 50.4 \pm 10.1$ respectively) and significantly $(\mathrm{P}<0.001)$ lower the TG levels (120.4 $\pm 29.59 \& 133.0 \pm 30.52$ respective-ly). Chen et al. (2013) reported that the longterm effects of PSI were associated with reduced TG and increased HDL-C in Chinese patients. A study in rural Indonesia showed that intestinal helminthes were negatively associated with lipid levels (Wiria et al, 2013). Another study also found significantly $(\mathrm{P}<0.001)$ lower plasma lipid levels in children (7-13 years) infected with $S$. haematobium compared with controls (Onuegbu et al, 2011). Dyslipidemia characterized by hypertrigly-ceridemia and low HDL-C was referred to an atherogenic dyslipidemia and frequently detected in patients with MS 
Table 5: Most associated components of MS with each group

\begin{tabular}{|c|c|c|c|c|c|c|c|}
\hline & & \multirow{2}{*}{$\mathrm{B}$} & \multirow{2}{*}{ Wald } & \multirow{2}{*}{$\mathrm{P}$} & \multirow{2}{*}{ OR } & \multicolumn{2}{|c|}{$95.0 \%$ C.I. for $\operatorname{EXP}(B)$} \\
\hline & & & & & & Lower & Upper \\
\hline \multirow{6}{*}{ 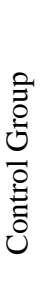 } & Sex(Female $)$ & 2.119 & 22.820 & $<0.001$ & 8.324 & 3.489 & 19.860 \\
\hline & $\mathrm{WC}$ & 0.036 & 12.905 & $<0.001$ & 1.036 & 1.016 & 1.056 \\
\hline & TG & 0.029 & 25.777 & $<0.001$ & 1.030 & 1.018 & 1.042 \\
\hline & HDL & 0.066 & 16.304 & $<0.001$ & 0.936 & 0.907 & 0.967 \\
\hline & HTN (Hypertensive) & 1.838 & 26.218 & $<0.001$ & 1.159 & 1.079 & 1.322 \\
\hline & FBS & 0.035 & 27.221 & $<0.001$ & 1.036 & 1.022 & 1.050 \\
\hline \multirow{6}{*}{ 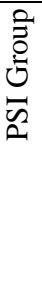 } & BMI & 0.547 & 11.250 & 0.001 & 1.728 & 1.255 & 2.378 \\
\hline & TG & 0.027 & 14.833 & $<0.001$ & 1.027 & 1.013 & 1.042 \\
\hline & HDL & 0.106 & 22.424 & $<0.001$ & 0.899 & 0.861 & 0.940 \\
\hline & HTN (hypertensive) & 2.915 & 33.274 & $<0.001$ & 1.054 & 1.020 & 1.146 \\
\hline & DBP & 0.065 & 6.922 & 0.009 & 1.067 & 1.017 & 1.119 \\
\hline & FBS & 0.055 & 31.062 & $<0.001$ & 1.057 & 1.037 & 1.078 \\
\hline
\end{tabular}

. Dyslipidemia was recently identified as a residual cardiovascular risk factor (Miller, 1998; Liberopoulos et al, 2005). Besides, dyslipidemia was found in systemic lupus erythematosus and rheumatoid arthritis (Sherer et al, 2010; Gonzalez-Gay and Gonzalez-Juanatey, 2012). Atherosclerosis is a chronic inflammatory and autoimmune disease, which were central to onset of the disease and progression (Ohashi et al, 2004; Mahmoudi et al, 2007). Moreover, plaque formation in atherosclerosis was considered to resemble the pathogenesis of other autoimmune diseases (Hansson, 2005). The mouse immune system was found to produce a strong anti-inflammatory response to help suppress the progression of arteriosclerosis (Subra-manian et al, 2013). The mechanism might be due to the immunomodulatory role induced by $S$. mansoni eggs in suppressing lipogenesis, and improving insulin sensitivity and glucose metabolism (Bhargava et al, 2012). Moreover, the S. mansonisoluble egg antigen (SEA) treatment induced the anti-inflammatory macrophages, attenuates atherosclerosis development and inhibits plaque necrosis.

The study showed that age, sex, height, weight, education, history of hypertens-ion, $\mathrm{Hb}$. level and serum level ALT did not significantly differ $(\mathrm{P}>0.05)$ when compared the PSI and control groups.
In this study, the FBG level was insignificantly lower $(\mathrm{P}>0.05)$ in PSI than control $(105.8 \pm 23.1 \& 107.8 \pm 26.3$ respectively). Chen et al. (2013) reported that participants with PSI had significantly lower levels of fasting blood glucose, postprandial blood glucose, as well as a lower prevalence of diabetes $(14.9 \%$ vs. $25.4 \%, \mathrm{P}<0.001)$ compared with the control. A study in China also found significantly lower FBG level in participants with PSI compared with those without PSI (Shen et al, 2015).

Du et al. (2014) reported that schistosomiasis patients with severe liver damage had statistically significant increase in the incidence of diabetes. It has been shown that $S$. mansoni soluble egg antigens can profoundly regulate the immune system of the infected host and to prevent type 1 diabetes in non-obese diabetic (NOD) mice. The splenocytes from soluble egg antigen-treated mice have reduced ability to transfer diabetes to NOD (Zaccone et al, 2003). Further studies showed a prolonged production of cytokines IL-10, IL-4, and interferon gamma (IFN $\gamma$ ), in schistosome egg-infected NOD mice. Previous studies have suggested that abnormal cytokine secretion participate in the pathophysiology of diabetes and its complications (Mooradian et al, 1991; Kung et al, 2010). Schistosome infection may affect glucose metabolism through long-term effect on the $\mathrm{T}$ cell related cytokine secre- 
tion. Inflammation and altered innate immunity have also been implicated in the pathogenesis of diabetes through insulin resistance (Wellen and Hotamisligil, 2005). Wu et al. (2011) had demonstrated elegantly that helminth induced adipose tissue eosinophilia enhanced glucose tolerance and improved insulin resistance in mice fed with a high fat diet. Moreover, although the parasite was cleared after 8 days, metabolic response was sustained.

In the present study, logistic regression analysis revealed statistically significant relative risk reduction in MS in PSI incl-uded central obesity, hypertriglyceridemia, low HDL-C, hypertension and hyperglycaemia when compared with control group. This finding was somewhat consistent with the cross sectional study conducted in Jiading, a suburban of Shanghai, China, which reported that PSI correlated to a low prevalence of diabetes \& MS (Chen et al, 2013). Shen et al. (2015) reported that logistic regression analysis revealed significant negative associations between PSI \& MS and its components.

\section{Conclusion}

The outcome data showed a protective effect of PSI against metabolic syndrome. This may be reconsidered in terms of therapeutic strategies, particularly in the design of vaccines and vaccine trials of schistosome for the treatment of metabolic diseases. Extensive study is ongoing to confirm this outcome result.

\section{References}

Alberti, KG, Zimmet, P, Shaw, J, 2005: The metabolic syndrome-a new worldwide definition. Lancet 366:1059-62.

Bhargava, P, Li, C, Stanya, KJ, Jacobi, D, Dai, L, et al, 2012: Immunomodulatory glycan LNFPIII alleviates hepatosteatosis and insulin resistance through direct and indirect control of metabolic pathways. Nat. Med. 18: 1665-72.

Chen, Y, Lu, J, Huang, Y, Wang, T, Xu, Y, et al, 2013: Association of previous schistosome infection with diabetes and metabolic syndrome: A cross-sectional study in rural China. J. Clin. Endocrinol. Metab. 98:E283-7.
Du, A, Yun, P, Zhong, W, Li, Y, Zeng, J, 2014: Analysis of blood glucose levels in patients with Schistosomiasis. BTAIJ 10, 12: 59514.

Gonzalez-Gay, MA, Gonzalez-Juanatey, C, 2012: Inflammation, endothelial function and atherosclerosis in rheumatoid arthritis. Arthrtis Res. Ther. 14:122-8.

Hansson, GK, 2005: Inflammation, atherosclerosis, and coronary artery disease. N. Engl. J. Med. 352:1685-95.

Kung, WJ, Lin, CC, Liu, SH, Chaung, HC, 2010: Association of interleukin- 10 polymorphisms with cytokines in type 2 diabetic nephropathy. Diabet.Technol. Ther. 12: 809-13

Liang, S, Yang, C, Zhong, B, Guo, J, Li, H, et al, 2014: Surveillance systems for neglected tropical diseases: global lessons from China'sevolving schistosomiasis reporting systems, 1949-2014. Emerg. Them. Epidemiol. 11:19-22.

Liberopoulos, EN, Daskalopoulou, SS, Mikhailidis, DP, 2005: Management of high tryglycerides: what non-specialists in lipids need to know. Hell J. Cardiol. 46: 268-72.

Liu, Z, Bing, X, Zhi, XX, 2002: Predictive values of body mass index and waist circumference to risk factors of related diseases in Chinese adult population: Cooperative meta-analysis Group of China Obesity Task Force 23:5-10.

Mahmoudi, M, Curzen, N, Gallagher, PJ, 2007: Atherogenesis: the role of inflammati-ons and infection. Histopathology 50:535-46.

Melon, A, Wang, A, Phan, V, McKay, DM, 2010: Infection with Hymenolepis diminuta is more effective than daily corticosteroids in blocking chemically induced colitis in mice. J. Biomed. Biotechnol. 9:384-423.

Miller, M, 1998: Is hypertriglyceridaemia an independent risk factor for coronary heart disease? the epidemiological evidence. Eur. Heart J. 19:H18-22.

Mooradian, AD, Reed, RL, Meredith, KE, Scuderi, P, 1991: Serum levels of tumor necrosis factor and IL-1and IL-1 in diabetic patients. Diabetes Care 14:63-5.

Mottillo, S, Filion, KB, Genest, J, Joseph, L, Pilote, L, et al, 2010: The metabolic syndrome and cardiovascular risk a systematic review and meta-analysis. J. Am. Coll. Cardiol. 56:1113-32.

Ohashi, R, Mu, H, Yao, Q, Chen, C, 2004: Atherosclerosis: immunopathogenesis and immunotherapy. Med. Sci. Monit. 10:255-60. Onuegbu, JA, Olisekodiaka, JM, Oladele, HA 
Opeyemi, US, Igbeneghu, CA, et al, 2011: Lipid profile of subjects infected with Schistosoma haematobium in South-Western Nigeria. Pakistan J. Med. Sci. 27:44-8.

Reddy, A, Fried, B, 2007: The use of Trichuris suis and other helminth therapies to treat Crohn's disease. Parasitol. Res. 100:921-7.

Reddy, A, Fried, B, 2009: An update on the use of helminths to treat Crohn's and other autoimmunune diseases. Parasitol. Res. 104: 217-21.

Richter, J, Christopher, H, Campagne, G, Bergquist, NR, Jenkins, JM, 2000: Ultrasound in schistosomiasis: A practical guide to the standardized use of ultrasonography for the assessment of schistosomiasis-related morbidity, Report of a WHO expert committee. Geneva, Switzerland.

Sengupta, S, 2008: Are metabolic indices different between drug-naïve first-episode psychosis patients and healthy controls? Schizophrenia Res. 102:329-36.

Shen, ZH, Xu, M, Yao, WF, Feng YB, Yun, JT, et al, 2015: The potential long-term effect of previous schistosome infection reduces the risk of metabolic syndrome among Chinese men. Parasite Immunol. 37:333-9.

Sherer, Y, Zinger, H, Shoenfeld, Y, 2010: Atherosclerosis in systemic lupus erythematosus. Autoimmunity 43:98-102.

Subramanian, M, Thorp, E, Hansson, GK, Tabas, I, 2013: Treg-mediated suppression of atherosclerosis requires MYD88 signaling in DCs. J. Clin. Invest. 123:179-88.

Von Moltke, J, Locksley, RM, 2014: I-L-C-2 it: type 2 immunity and group 2 innate lymphoid cells in homeostasis. Curr. Opin. Immunol. 31C:58-65.

Wellen, KE, Hotamisligil, GS, 2005: Inflammation, stress, and diabetes. J. Clin. Invest. 115:1111-9.

Wiria, AE, Sartono, E, Supali, T, Yazdanbakhsh, M, 2014: Helminth infections, type-2 immune response, and metabolic syndrome. PLoS Pathog. 10, e1004140

Wiria, AE, Wammes, LJ, Hamid, F, Dekkers, OM, Prasetyani, MA, et al, 2013: Relationship between carotid intima media thickness and helminth infections on Flores Island, Indonesia. PLoS One 8:e548-55.

Wu, D, Molofsky, AB, Liang, HE, RicardoGonzalez, RR, Jouihan, HA, et al, 2011: Eosinophils sustain adipose alternatively activated macrophages associated with glucose homeostasis. Science 332:243-7.

Zaccone P, Fehervari Z, Jones FM, et al, 2003: Schistosoma mansoni antigens modu-late the activity of the innate immune response and prevent onset of type 1 diabetes. Eur J Immunol. 33:1439-49.

Zhu, YC, Wu, Gl, Guan, XH, 2008: Immunology of schistosome infection [in Chinese] Shanghai: Shanghai Scientific and Technical Literature Publishing House. 